\title{
The Application Research of 3D Simulation Modeling Technology in the Sports Teaching YANG Jun-wa ${ }^{1, a}$ \\ ${ }^{1}$ Zhengde Polytechnic College, Nanjing 211106, China \\ a yangjunwa@qq.com
}

\section{Keywords: Sports Teaching; Three-Dimensional; Simulation Modeling}

\begin{abstract}
With the current development of information technology in sports training in computer-aided training has become increasingly common to achieve a sports training video feedback analysis system, through pattern recognition, image processing, video analysis and other modern computer technology information processing technology the sports training and computer application technology, computer vision from the perspective of analysis and study of sports. Based on three-dimensional modeling of motion simulation can help sports training, sports science analysis methods to achieve experiential training methods from stylized transition, leading to faster and more effectively improve the level of sports training and athletic performance. The use of a simplified model of the human skeleton, in conjunction with specific prior knowledge of sports action, resulting in partial attitude information athletes. Design and implementation of information technology systems will be combined with physical training, can give a variety of auxiliary information sports action, and can effectively assist coaches and athletes to improve training efficiency.
\end{abstract}

\section{Introduction}

Sports simulation system can be further combined sports full experience of sports action for analysis, to identify the type of action, extracting the outline of athletes, athletes' posture preliminary analysis. Real-time processing part of the system, mainly to obtain prospects in part by video motion background separation technology to remove all kinds of noise, obtained in which part of the video player [1-2]. Then centroid approximate center of gravity, the center of gravity trajectory fit athlete, combined with camera calibration parameters in advance, calculate important parameters coaches and athletes concerned, the use of voice and on-screen display a variety of ways, instant feedback to coaches and athlete. The system also can be for some sports action for further analysis and processing [3].

Due to the development of computer technology, increasing data storage capacity and rapid decline in prices, resulting in data storage athletes is a quick and cheap. How to use these large amounts of data, in-depth analysis of technical movement essentials, athletes, and coaches training provided useful reference information [4]. Therefore, the application of modern computer technology has become the most important tools to improve the level of competition. An important reason for the majority of sports projects did not successfully develop simulation training system is collaborative approach sports training coaches and athletes and computer software developers is not reasonable, we need to study the characteristics of professional sports training and computer simulations to develop the simulation system according to Sports Training Most problems have developed simulation software accuracy, practical aspects;; model; many coaches do not fully understand the role of simulation training software that has been developed is difficult to truly meet the needs of end users, software development needs analysis done early Did not do well. .

\section{Human joint model overview}

The human skeleton model is the base model of the human body. Important because it contains the joint three-dimensional location information, it can better represent the human information. 
Artificial joint model used in Figure 1, which contains a total of 23 joints, the root of the waist. Because doing so would cause motion capture coordinate data is based on the world coordinate system, we first will coordinate every action will be converted to the root of the coordinate origin of the world coordinate system, in order to make action sequences absolute position data regardless of the body [5-6].

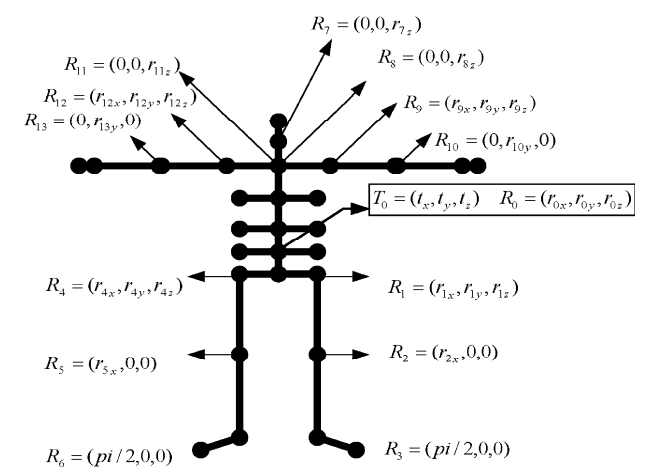

Figure1. 3D human skeleton model

$$
\begin{aligned}
& M_{P_{x} C}=T\left(t_{x}, t_{y}, t_{z}\right) \cdot R_{z}(\gamma) \cdot R_{y}(\beta) \cdot R_{x}(\alpha) \\
& T\left(t_{x}, t_{y}, t_{z}\right)=\left[\begin{array}{llll}
1 & 0 & 0 & t_{x} \\
0 & 1 & 0 & t_{y} \\
0 & 0 & 1 & t_{z} \\
0 & 0 & 0 & 1
\end{array}\right] \\
& R_{x}(\alpha)=\left[\begin{array}{cccc}
1 & 0 & 0 & 0 \\
0 & \cos (\alpha) & -\sin (\alpha) & 0 \\
0 & \sin (\alpha) & \cos (\alpha) & 0 \\
0 & 0 & 0 & 1
\end{array}\right] \\
& R_{y}(\beta)=\left[\begin{array}{cccc}
\cos (\beta) & 0 & \sin (\beta) & 0 \\
0 & 1 & 0 & 0 \\
-\sin (\beta) & 0 & \cos (\beta) & 0 \\
0 & 0 & 0 & 1
\end{array}\right] \\
& R_{z}(\gamma)=\left[\begin{array}{cccc}
\cos (\gamma) & -\sin (\gamma) & 0 & 0 \\
\sin (\gamma) & \cos (\gamma) & 0 & 0 \\
0 & 0 & 1 & 0 \\
0 & 0 & 0 & 1
\end{array}\right]
\end{aligned}
$$

For any child node point $p=(\mathrm{x}, \mathrm{y}, \mathrm{z}, 1)^{\mathrm{T}}$ within the local coordinate system, the position within the parent node of the local coordinate system is $P=(\mathrm{X}, Y, \mathrm{Z}, 1)^{\mathrm{T}}$.

$$
\left[\begin{array}{c}
X \\
Y \\
Z \\
1
\end{array}\right]=\left[\begin{array}{llll}
1 & 0 & 0 & t_{x} \\
0 & 1 & 0 & t_{y} \\
0 & 0 & 1 & t_{z} \\
0 & 0 & 0 & 1
\end{array}\right]\left[\begin{array}{cccc}
\cos (\gamma) & -\sin (\gamma) & 0 & 0 \\
\sin (\gamma) & \cos (\gamma) & 0 & 0 \\
0 & 0 & 1 & 0 \\
0 & 0 & 0 & 1
\end{array}\right]\left[\begin{array}{ccccc}
\cos (\beta) & 0 & \sin (\beta) & 0 \\
0 & 1 & 0 & 0 \\
-\sin (\beta) & 0 & \cos (\beta) & 0 \\
0 & 0 & 0 & 1
\end{array}\right]\left[\begin{array}{cccc}
1 & 0 & 0 & 0 \\
0 & \cos (\alpha) & -\sin (\alpha) & 0 \\
0 & \sin (\alpha) & \cos (\alpha) & 0 \\
0 & 0 & 0 & 1
\end{array}\right]\left[\begin{array}{c}
x \\
y \\
z \\
1
\end{array}\right]
$$

This chain through exercise, to express the relationship between the coordinates of each point with each rotation angle, the position of each point in the world coordinate system can be determined.

At the same time, also to the initial positioning of the body z-axis positive direction, so that the initial orientation has nothing to do with human data. At the same time, we will get different people bones length normalized to the same size made independent of the size of the skeleton and human motion data [7]. This can be unified into a standard coordinate joint race, valid data dimension 67-dimension (cross-sectional information including root We also height information is broken down into a series of human bone parts, each part is a combination of single-joint or joints. point. Such a node classification contains semantic information, so we operate more efficiently extract a key part of the identification. 


\section{Build sports technical movement features of the model}

In order to achieve integrity and the independence movement database design needs, we need to realize that the correct classification of technical movements, but in order to achieve common needs, we need to ensure that actions are scalable library. According to the motion operation of the technical action task decomposition model and classification methods, we propose a hierarchy Players libraries, from top to bottom can be divided into action unit layer, layer, layer, action elements and technical operations, shown in Figure 2 .

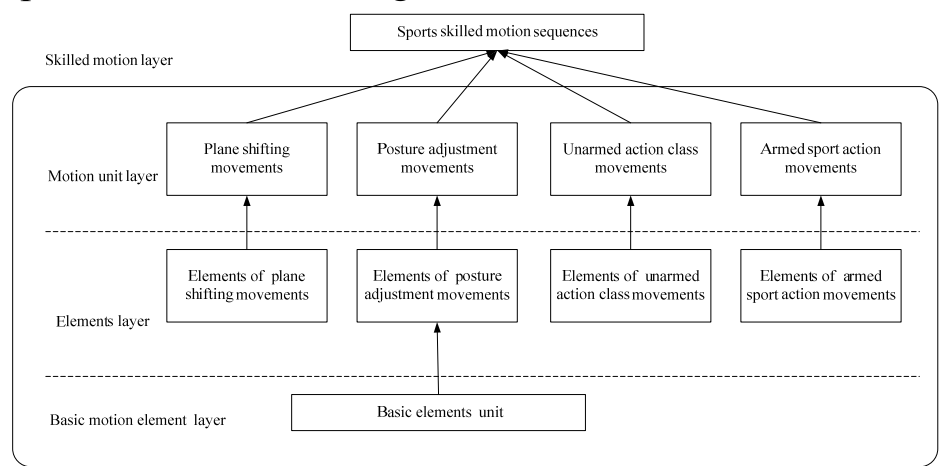

Figure 2.The hierarchical model of sports skilled motion characteristic

The basic action elements layer: This layer contains only one basic unit. The basic elements of a framework for action defines the basic action sports athletes, and describes some of the basic elements of relevant, including start-up operation cycle, pause, stop, and general motion parameters.

Technical action elements layer: Basic operating unit derived elements obtained technical action elements, and the basic semantic features of sports action, but the action can not be broken down semantics is human action sports most basic units. Sports action elements generally follow the model divided the human joints and freedom of movement.

Action cell layer: technical action unit is composed of several technical action elements in accordance with certain semantic rules constitute a certain degree of semantic features, describes the technical operations knowledge semantic features, but does not include any specific operational semantics related activities.

\section{D simulation modeling sport Training achieve}

3D sports training includes a three-dimensional motion simulation to achieve, the actual situation contrast, action choreography action editor, kinematics analysis, video analysis and other six auxiliary training methods, the upgrade version behind, will join Dynamics. These methods can help athletes understand and master the basic action sports to help athletes understand the nature of sports action from physiological and physical laws, according to their own conditions, the development of appropriate training measures. Actual comparison module standard action motion video and motion simulation system virtual athlete will be the same screen contrast viewing angle display, intuitive and accurate comparison of the difference between the two, to help athletes improve athletic performance and training efficiency. Three-dimensional motion analysis technology analysis including kinematic and dynamic analysis. As the rotation angle of the joint analysis of trends, analyzes different heights, athletes and other differences of the same length as the joint action, to quantify the state of image motion manner athletes displayed. We chose virtual athletes focus joint, the center of gravity of the visual display in a certain time trace shown in Figure 3.

System is based on human animation blending real-time motion redirection: First of a three-dimensional skeleton connection diagram automatically extracting three-dimensional model. Then some preprocessing operations skeleton model, so you can complete skeleton joint CMU-one match point; real-time automatic data collection Kinect motion-driven three-dimensional model, combining sports redirection techniques to ensure human characteristics of reality. Integrated system dual-threaded real-time animation design, shown in Figure 4. The first thread to access 
real-time motion capture human motion capture device data. The second thread is responsible for loading $3 \mathrm{~d}$ mesh, 3D mesh models and key point matching skeleton automatic extraction, skin deformation and motion redirection.

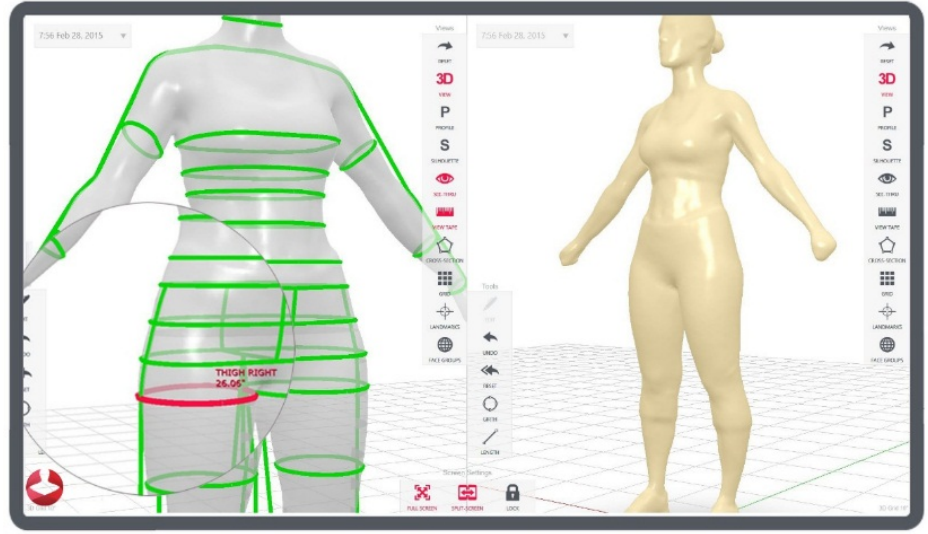

Figure 3. 3D modeling for virtual athletes

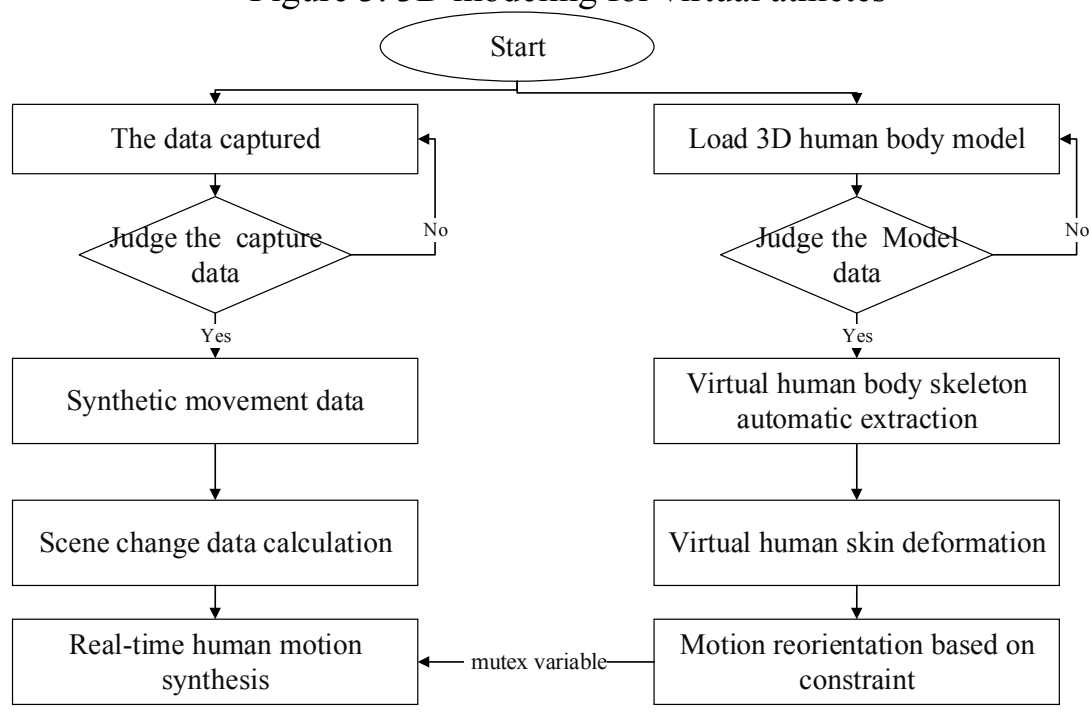

Figure 4. The design flow of three-dimensional sports training system

\section{Conclusion}

In order to maximize our human potential, modern sports science and technology needs to continue to intervene, which requires the integrated use of sports-related scientific disciplines of knowledge, such as the use of system simulation approach inherent law school sports conduct research. In recent years, the system simulation research focus has object-oriented simulation method qualitative simulation, distributed interactive simulation, visualization, simulation, multimedia simulation and intelligent simulation. With the development of three-dimensional computer technology, computer simulation of human motion analysis in sports It has been widely used. The establishment of human movement model is the key to conduct computer simulations of human movement. However, modeling is a difficult and complex task, the traditional method has its own characteristics and advantages, but also has its limitations. In this paper, 3D modeling and simulation-based technology for sports training aid approach. By modeling the movement of real athletes, so that the virtual athlete to accurately reproduce the gymnastic athletes to help athletes improve technical movements, improve the technical level.

\section{Reference}

[1] George A P, De R. Review of temporal bone dissection teaching: how it was, is and will be[J]. The Journal of Laryngology \& Otology, 2010, 124(02): 119-125. 
[2] Schlick T. Molecular modeling and simulation: an interdisciplinary guide: an interdisciplinary guide[M]. Springer Science \& Business Media, 2010.

[3] Kataoka R, Ebisuzaki T, Kusano K, et al. Three - dimensional MHD modeling of the solar wind structures associated with 13 December 2006 coronal mass ejection[J]. Journal of Geophysical Research: Space Physics (1978-2012), 2009, 114(A10).

[4] Tajima K, Chen K K, Takahashi N, et al. Three-dimensional finite element modeling from CT images of tooth and its validation[J]. Dental materials journal, 2009, 28(2): 219-226.

[5] Ahmed K, Keeling A N, Fakhry M, et al. Role of virtual reality simulation in teaching and assessing technical skills in endovascular intervention[J]. Journal of Vascular and Interventional Radiology, 2010, 21(1): 55-66.

[6] Xiang Y, Arora J S, Abdel-Malek K. Physics-based modeling and simulation of human walking: a review of optimization-based and other approaches[J]. Structural and Multidisciplinary Optimization, 2010, 42(1): 1-23.

[7] Waran V, Narayanan V, Karuppiah R, et al. Injecting realism in surgical training-initial simulation experience with custom 3D models[J]. Journal of surgical education, 2014, 71(2): 193-197. 